\title{
Incidence and Severity of Huanglongbing and Candidatus Liberibacter asiaticus Titer among Field-infected Citrus Cultivars
}

\author{
Ed Stover ${ }^{1}$ and Greg McCollum \\ USDA, ARS, US Horticultural Research Laboratory, 2001 S. Rock Road, \\ Ft. Pierce, FL 34945
}

Additional index words. greening, resistance, tolerance, PCR

\begin{abstract}
Incidence and severity of Huanglongbing (HLB) disease were assessed in Apr. 2010 among eight citrus cultivars representing diverse scion types growing in commercial groves in Florida's Indian River region, an area with a high incidence of HLB. In each grove, 20 trees of each cultivar were rated for visual HLB symptoms and leaves were collected for quantitative polymerase chain reaction quantification of Candidatus Liberibacter asiaticus (CLas), the presumptive causal agent of HLB. There was a strong correlation between HLB rating and CLas titer (titer represented by $\mathrm{Ct}, r^{2}=0.37$ and 0.40 , for whole tree and leaf sample, respectively, both with $P<0.0001$ ) across all cultivars and groves. Although incidence and severity of HLB varied considerably among the groves, scion-specific differences were apparent, even when analyses excluded potentially confounding grove effects. 'Temple' tangor showed the most consistently low incidence of HLB symptoms and CLas titer; in contrast, 'Murcott' tangor and 'Minneola' tangelo had the highest incidence of HLB symptoms and highest CLas titer. These results suggest useful resistance to HLB with reduced symptoms and reduced CLas titer may be found in conventional scion cultivars and further work is needed to assess this potential and its commercial value.
\end{abstract}

Huanglongbing is a very destructive citrus disease (Bove, 2006) and is currently considered to be the greatest threat facing the Florida and U.S. citrus industry (Gottwald, 2010). HLB is associated with a phloem-limited bacterium, CLas, and its insect vector, Diaphorina citri, the Asian citrus psyllid (ACP). As HLB progresses, trees become increasingly debilitated, leading to loss of yield and poor fruit quality followed eventually by tree death (Bove, 2006; Gottwald, 2010). Since HLB was first confirmed in Florida in 2005, it has now been found in all 32 citrus-producing counties in the state as well as in dooryard citrus in non-citrus-producing counties (Halbert et al., 2008).

Currently, the strategies for management of HLB include insecticide applications for vector control, roguing trees affected by the disease to reduce inoculum, and production and use of nursery stock free of CLas/ACP (Gottwald, 2010). Insecticide applications for ACP control have escalated in Florida increasing the likelihood of ACPs developing insecticide resistance (e.g., Srinivasan et al., 2008). Scouting to identify trees expressing HLB symptoms and subsequent tree removal are costly and reduce fruit yield with each

Received for publication 14 July 2011. Accepted for publication 27 July 2011.

The technical assistance of Gregory Brock, Angela Ledger, and Luc Overholt is gratefully acknowledged. ${ }^{1}$ To whom reprint requests should be addressed; e-mail ed.stover@ars.usda.gov. tree removed. The ability to grow replanted trees near HLB-affected groves is uncertain as a result of the presence of inoculum and frequent initiation of new leaf growth by young trees, which attracts ACP (Keiji et al., 2005). A more sustainable approach for the management of HLB would be the cultivation of citrus cultivars that are unaffected, or less affected, by the disease rather than more susceptible types (Gottwald, 2010).

Several studies have been conducted to determine if there are differences in HLB susceptibility among citrus cultivars and/or species. Compared with other tested cultivars within individual experiments, lower susceptibility to HLB associated with CLas has been reported for limes (de Lange et al., 1985; Schwarz et al., 1973; Shokrollah et al., 2009), pummelos (Koizumi et al., 1997; Schwarz et al., 1973), lemons (Cheema et al., 1982; Nariani, 1982; Schwarz et al., 1973), some mandarin types (e.g., 'Ladu' and 'Som Pan' in Thailand; Koizumi et al., 1997), and various non-cultivated citrus or related species. However, reports are inconsistent which may reflect genotypes selected, interactions between the host genotype and strains of HLB pathogens studied (e.g., Kiritani and $\mathrm{Su}$, 1999), or the methods used to evaluate disease. Reports before routine use of molecular diagnostic tools may also reflect confusion of apparent HLB with other diseases. Poncirus trifoliata (proposed as Citrus trifoliata in a recent revision of citrus taxonomy; Bayer et al., 2009) appears to be less susceptible to HLB than are cultivated citrus scion varieties.
P. trifoliata and its hybrid, 'Carrizo' citrange, developed less severe HLB symptoms and among the lowest titer of CLas among the genotypes evaluated in a recent greenhouse study (Folimonova et al., 2009). This is strong evidence that variation for HLB susceptibility exists within the citrus gene pool, and it is possible that scion cultivars may display useful resistance.

The majority of citrus production in Florida is represented by sweet orange ( $C$. sinensis, $\approx 86 \%$ of the $\approx 200,000$ ha of Florida citrus) followed by grapefruit (C. paradisi, $10 \%$ of total Florida citrus; NASS, 2008). In the Folimonova et al. (2009) study on citrus susceptibility to HLB, sweet orange was in the most susceptible category and grapefruit showed slightly lower susceptibility. "Other citrus" grown in Florida is mostly mandarin hybrids (tangors, tangerines, and tangelos), which represent $\approx 9300$ ha or $4 \%$ of all Florida citrus (NASS, 2008). Tangors are natural hybrids of $C$. reticulata and $C$. sinensis; tangelos are hybrids between $C$. reticulata and C. paradisi; whereas tangerines range from pure $C$. reticulata to complex hybrids that have been created by plant breeders and have a phenotype similar to $C$. reticulata. There is much greater genetic diversity among the mandarin group than sweet oranges and grapefruit, which are each represented essentially by bud sports from single genotypes.

Although greenhouse studies using graft inoculation have been conducted to compare susceptibility to HLB among cultivars, it is unlikely that greenhouse results can be extrapolated to ultimate tree performance in the field. Replicated field studies of HLB susceptibility require years to complete. This study was conducted to detect differences in HLB severity and incidence as well as CLas titer among diverse citrus cultivars in extant field plantings known to be affected by HLB to provide guidance in selection of cultivars for long-term field tests.

\section{Materials and Methods}

Plant material. Our objective was to quantify the incidence and severity of HLB among citrus types growing in commercial groves with disease pressure typical of the region. Because Florida citrus production is dominated by sweet orange and grapefruit, there are few groves with diverse cultivars. As a consequence, it was impossible to develop a balanced sample set with all scions represented in all groves. The eight commercial citrus groves selected for survey each contained four or more scions (Table 1) planted within $1 \mathrm{~km}$ of each other. Groves were located in the Florida counties of Indian River, St. Lucie, Martin, and Palm Beach (lat. 26.7 to $27.6^{\circ} \mathrm{N}$ and long. 80.2 to $80.4^{\circ} \mathrm{W}$ ). Trees in each grove were mature bearing trees on standard industry rootstocks and had been maintained using local commercial practices regarding fertilization, irrigation, and pest control. Citrus scions evaluated in this study included sweet orange (cultivars Hamlin, Valencia, and navel), grapefruit ('Marsh', 'Ruby Red', and 'Flame'), tangors ('Temple', 
Table 1. Percentage of trees ( 20 samples per cultivar per grove) with one or more CLas genomes per $100 \mathrm{ng}$ nucleic acid sample from diagnostic leaf extraction as assessed in quantitative polymerase chain reaction. ${ }^{\mathrm{z}}$

\begin{tabular}{llcccccccc}
\hline & & \multicolumn{1}{c}{ Grove } \\
\cline { 3 - 10 } Type & Cultivar & 1 & 2 & 3 & 4 & 5 & 6 & 7 & 8 \\
\hline Sweet orange & C. sinensis & 45 & 20 & NA & 56 & 20 & 30 & 0 & 0 \\
Grapefruit & C. paradisi & 20 & 45 & 5 & 55 & NA & 5 & 0 & 0 \\
Tangor & Murcott & NA & 70 & NA & 65 & 5 & NA & NA & NA \\
Tangor & Temple & 10 & NA & 70 & 10 & 5 & 0 & 0 & 5 \\
Tangor & Ortanique & NA & NA & NA & NA & NA & NA & 30 & NA \\
Tangelo & Minneola & 55 & NA & 90 & 50 & 25 & 30 & 5 & 0 \\
Mandarin hybrid & Fallglo & 25 & NA & 20 & NA & NA & NA & NA & NA \\
Mandarin hybrid & Sunburst & 0 & 35 & NA & NA & NA & NA & 0 & NA \\
\hline
\end{tabular}

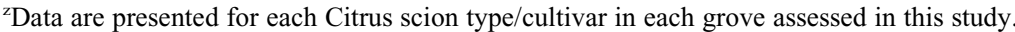

CLas $=$ Candidatus Liberibacter asiaticus; NA = not available.

'Murcott', and 'Ortanique'), and mandarin-type hybrids ('Fallglo', 'Minneola', and 'Sunburst') (Table 1). In each grove, 20 trees per cultivar were evaluated; however, in no case were all cultivars available in all groves (Table 1). A total of 720 trees were included in the study. Because there tends to be a higher than expected incidence of HLB-affected trees at the periphery of plantings (Gottwald et al., 2008), only trees more than three trees from the ends of rows and sides of blocks were considered for evaluation; at least three trees separated individual targeted trees. Target trees were identified randomly by row and tree before arrival at each grove.

Huanglongbing symptom rating. Targeted trees within the eight groves were surveyed for visual symptoms of HLB during Apr. 2010 with HLB severity rated using a 3-point scale $(0,1,2)$ with 0 representing no apparent HLB symptoms, 1 suspect HLB symptoms, and 2 likely HLB symptoms. Incidence of HLB was calculated by summing the number of trees rated 1 (suspect) or 2 (likely) and dividing the sum by the total number of trees.

Leaf sampling. Leaf samples for quantification of CLas were collected as visual rating was conducted. For each target tree, a sixleaf "most HLB symptomatic" sample was collected; for trees with no overt HLB symptoms, leaves of similar age and position as those selected based on symptoms were collected. Leaf samples were also rated for HLB symptoms using the 3-point scale described previously.

DNA isolation and Candidatus Liberibacter asiaticus quantification. Nucleic acid was extracted from citrus leaf laminar discs $(3 \mathrm{~mm}$ diameter, $\approx 11 \mathrm{mg}$ ) using REDExtract-NAmp (Sigma, St. Louis, MO) according to the manufacturer's instructions. Copy number of CLas 16S rDNA in citrus leaf nucleic acid extracts was determined using quantitative polymerase chain reaction (qPCR) following the standard protocol ( $\mathrm{Li}$ et al., 2006) and $\mathrm{Ct}$ values were converted to CLas copy number based on a standard curve generated in our laboratory $(\log$ Copy Number $=10.9-$ $0.27 \mathrm{Ct}$ ). Three copies of the $16 \mathrm{~s}$ rDNA gene are present in the CLas genome (Duan et al., 2009), and data were expressed as numbers of CLas genomes per gram of tissue, hereafter referred to as "CLas titer." Each sample was assayed in triplicate; positive, negative, and no template controls were included in each qPCR run.
Data analysis. Data were analyzed using SAS (Cary, NC) with all sweet orange cultivars combined in one group and all grapefruit cultivars combined in another group. Analyses included $\mathrm{Ct}$ values from $\mathrm{qPCR}$ (with a $\mathrm{Ct}$ value of 40 assigned when there was no detectable amplicon replication), grove-normalized $\mathrm{Ct}$ (an assessment in which the average $\mathrm{Ct}$ for each grove was subtracted from each tree's $\mathrm{Ct}$ value), $\mathrm{Ct}$ value only when $16 \mathrm{~s}$ rDNA amplification was detected, HLB severity rating on a 3-point scale, and percent of trees of each cultivar scoring 1 or 2 on the 3-point HLB scale. Mean and SE were calculated for parameters in each grove and cultivar. No standard data transformation resulted in Ct-derived data satisfying assumptions for analysis of variance, so means were compared using nonparametric analyses (Kruskal-Wallis when comparing more than two data sources and Wilcoxon for comparison of two cultivars in a one-sided $t$ test approximation) primarily comparing cultivars only when they were present in the same groves. Correlation between $\mathrm{Ct}$ value of samples and HLB rating of trees was conducted using Excel Analysis Tools (Microsoft Corp., Redmond, WA).

\section{Results and Discussion}

The objective of our study was to determine if differences in the incidence and severity of HLB, along with differences in CLas titer, could be detected among citrus types. We evaluated HLB in eight citrus scion types growing in commercial groves affected by HLB in the Indian River district of Florida. No cultivar displayed a consistently low or high level of CLas titer in every grove sampled (Table 1). Two methods, visual rating and quantification of CLas titer, were used to compare HLB among the scions.

Differences between cultivars in Candidatus Liberibacter asiaticus titer and visual Huanglongbing symptoms across all data. Based on the overall means $(\mathrm{n}=720)$ for HLB incidence (37\%) and CLas titer (mean $\mathrm{Ct}$ of 37.7) across all groves surveyed, most cultivars fall into one of two distinct groups (Fig. 1 A). Cultivars Temple, grapefruit, and Fallglo comprise a group with low HLB incidence and low CLas titer (high Ct values). Cultivars Ortanique, Minneola, and Murcott comprise a group that has HLB incidence greater than the overall mean and higher CLas titer (Ct values less than the overall mean). Sweet orange is intermediate among cultivars tested. This analysis does not consider the influence of individual grove differences in HLB incidence and cultivar composition, which are potentially confounding in analyzing overall means.

Differences between cultivars in Candidatus Liberibacter asiaticus titer after adjusting for grove differences. Because individual groves varied greatly in CLas titer (Fig. 1A), it is possible that distribution of cultivars across groves may influence apparent susceptibility to CLas. When $\mathrm{Ct}$ for each tree was normalized by individual grove means ("groveadjusted Ct all" in Fig. 2A; 'Ortanique' is omitted from this analysis because presence in a single grove resulted in a large $\mathrm{SE}$ ), 'Temple', 'Fallglo', 'Sunburst', and grapefruit show markedly lower CLas titers (higher relative $\mathrm{Ct}$ ) compared with other cultivars, whereas 'Minneola' and 'Murcott' showed substantially higher CLas titers. Mean separations between cultivars are similar for $\mathrm{Ct}$ values with and without normalization to grove means ("grove-adjusted Ct all" in Fig. 2A and "Ct all" in Fig. 2B). It is particularly significant that sweet orange, which is widely viewed as highly susceptible to HLB, is revealed as having an intermediate CLas titer notably lower than some cultivars surveyed with a higher Ct than 'Minneola' and 'Murcott'.

Differences between cultivars in Candidatus Liberibacter asiaticus titer only when amplicon was detected. Potential components of HLB resistance include reduced probability of initial infection and reduced proliferation of CLas once infection occurs. When CLas titers were compared only in trees in which the target CLas sequence amplified ("Ct amp only" in Fig. 2B), the cultivar separations for mean CLas titer (analyzed as Ct values) were similar to the separations for cultivar means across all trees including those with no CLas ("Ct all" in Fig. 2B).Therefore, 'Temple' and 'Fallglo' display lower CLas titers than 'Minneola', 'Murcott', and sweet orange even when comparing only those trees that do display infection. This observation is consistent with either reduced likelihood of 'Temple' and 'Fallglo' becoming initially infected, so that infections begin later but progress at the same rate after initiation, or reduced proliferation once infection occurs.

Differences between cultivars in Candidatus Liberibacter asiaticus titer comparing cultivars only in the same groves. Results of nonparametric analyses on CLas titer are presented in Table 2. Although it was not possible to develop a balanced sample set with all scions of interest represented in all groves, a subset of cultivars (sweet orange, grapefruit, Temple, and Minneola) were all present in each of five groves and provided a balanced set of samples. Among the cultivars that comprised this balanced sample set, 'Temple' had the lowest CLas titer; grapefruit was intermediate in CLas titer; whereas sweet orange and 'Minneola' had the highest titers (mean separations in Table 2 footnotes). Groves 1 , $4,6,7$, and 8 , which contain each of these four 

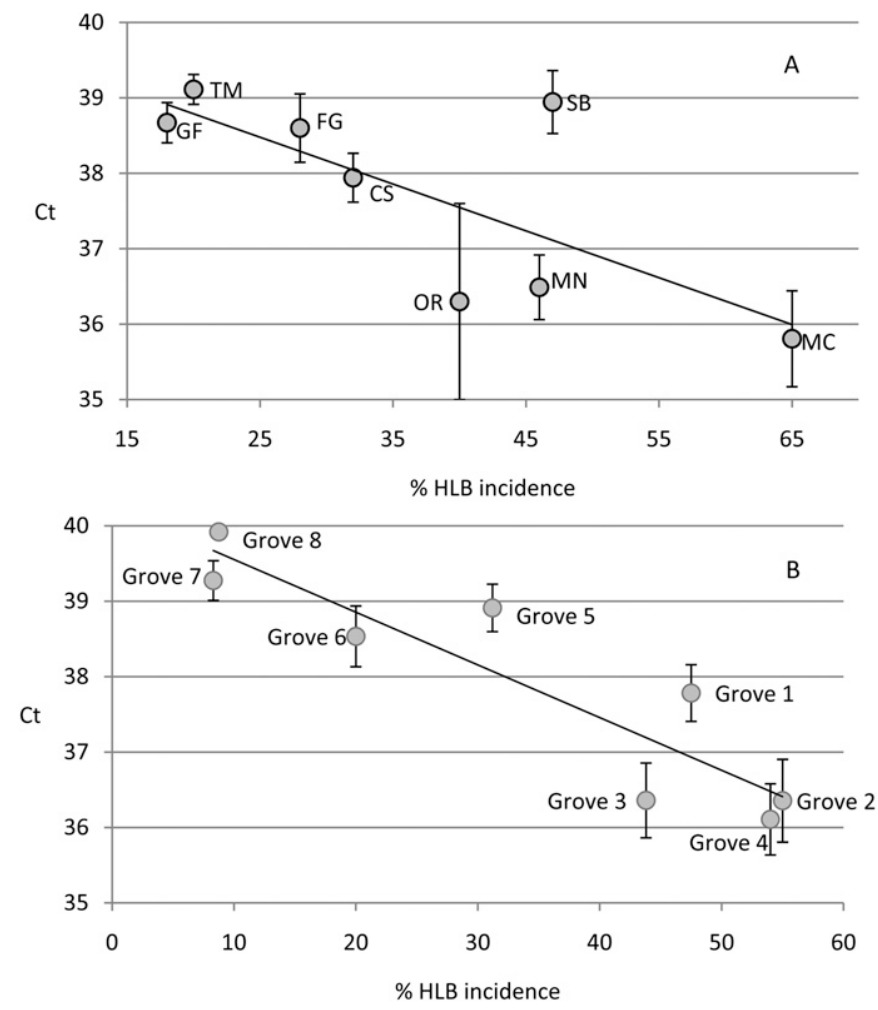

Fig. 1. Correlation of visual Huanglongbing (HLB) incidence (percent of trees scored as possible or likely HLB) with Ct on a scion cultivar (A) or grove (B) basis. A Ct of 40 was assigned to an individual tree if no amplification occurred. Letters next to data points in $\mathbf{A}$ are cultivar identity (CS = Citrus sinensis; $\mathrm{FG}=$ 'Fallglo'; $\mathrm{GF}=$ grapefruit; $\mathrm{MN}=$ 'Minneola'; $\mathrm{MC}=$ 'Murcott'; $\mathrm{SB}=$ 'Sunburst'; TM = 'Temple'), whereas numbers adjacent to data points in $\mathbf{B}$ indicate grove identity using numerical designations also used in Table 1. Each data point represents the mean $\mathrm{Ct}$ value $\pm \mathrm{SE}$.

cultivars, represent the full spectrum of HLB incidence on a grove by grove basis; Groves 7 and 8 had low incidence of HLB $(\approx 10 \%)$, whereas Groves 1 and 4 had high HLB incidence ( $45 \%$ to $55 \%)$. Although this balanced set of groves varied markedly in mean CLas titer $(P<0.0001)$ across all tested trees, when comparisons were made only in trees in which target CLas sequence amplified, there was no significant difference between mean CLas titer in groves $(P=0.4167)$, whereas differences in CLas titer between cultivars were more marked $(P=0.0674)$.

Nonparametric analyses were conducted between each pair of cultivars present together in three or more groves comparing data only within trees of those cultivars in shared groves (Table 2). 'Murcott' $(P=0.0298)$ displayed higher CLas titer than sweet orange. 'Temple' had significantly lower CLas titer than sweet orange $(P<0.0001)$ and 'Minneola' $(P<$ $0.0001)$, whereas grapefruit had lower CLas titer than 'Minneola' $(P<0.0001)$. These data are fairly consistent with overall trends depicted in Figure 1 in which means were analyzed for cultivars across all groves surveyed.

When CLas titer was compared only in trees in which the CLas 16s rDNA amplified (Table 2), 'Minneola' had a higher titer than sweet orange $(P=0.0113)$, 'Temple' $(P=$ $0.0039)$, and grapefruit $(P=0.0665)$. 'Murcott' had higher CLas titer than sweet orange $(P=$ $0.0113)$. In these comparisons, 'Temple' had lower CLas titer than 'Minneola' $(P=0.0039)$ and grapefruit $(P=0.0197)$. These data suggest the possibility that CLas titers may vary markedly between cultivars even when infection does occur, which may be significant in development of the HLB epidemic.

Apparent Huanglongbing resistance in this study compared with previous reports. Results of our study are somewhat consistent with previous reports on the same cultivars. Based on severity of symptoms and the ability of plants to continue growth after inoculation, 'Minneola' was reported to be one of the genotypes most susceptible to HLB in a study using graft-inoculated trees in the greenhouse (Folimonova et al., 2009). In the same study it was also reported that 'Valencia' sweet orange and 'Duncan' and 'Ruby Red' grapefruit were equivalent to 'Minneola' in terms of CLas titers in symptomatic HLB-symptomatic leaves, but these trees were graft-inoculated rather than being infected through ACP. Anecdotal reports based on observations in Florida suggest that 'Minneola' is especially susceptible to HLB, sweet orange very susceptible, and grapefruit somewhat more resistant. 'Minneola' is also among the most susceptible cultivars to the greening disease caused by Candidatus L. africanus in South Africa (H. le Roux, personal communication).

Relationship between Huanglongbing severity and Candidatus Liberibacter asiaticus titer. It has been previously reported that graftinoculated sweet orange display HLB foliar symptoms that correlate with CLas titer
(Coletta-Filho et al., 2010). To our knowledge, no one has explored this relationship within a group of cultivars. The relationship between ratings of HLB severity and CLas (analyzed as $\mathrm{Ct}$ values) in citrus leaf nucleic acid extracts is presented in Table 3. Whether expressed as whole-tree HLB severity rating or as leaf sample rating, there was a strong correlation between rating and CLas titer (titer is represented by $\mathrm{Ct}$ value, which is logarithmically and negatively correlated with genome copy number, $r^{2}=0.37$ and 0.40 , for whole tree and leaf sample, respectively, both with $P<0.0001$ ). Leaf sample ratings of 0,1 , or 2 correspond with $\mathrm{Ct}$ values of $39.6,37.8$, and 32.8 , respectively.

Although estimates of CLas titer in the current study are low (suspect HLB $=10^{2}$ to $10^{3}$ and likely HLB $=10^{3}$ to $10^{4}$ genomes $/ \mathrm{g}$ tissue, respectively), they fall within the range of $10^{3}$ genomes/g tissue (Coletta-Filho et al., 2010 ) and $10^{5}$ genomes $/ g$ tissue (Trivedi et al., 2009) reported for infected but asymptomatic tissue. Coletta-Filho et al. (2010) indicated that HLB symptoms begin to appear when the titer of CLas is greater than $10^{4}$ to $10^{5}$ genomes/g tissue, 10 -fold higher than the titer we found present in leaves rated as likely HLB-symptomatic, whereas full blotchy mottle symptoms appeared at $10^{6}$ to $10^{7}$ genomes $/ \mathrm{g}$ tissue. As a result of the emergence of the opportunity to conduct this study and its perceived urgency, we collected samples during the spring, the time when CLas titers as well as visible HLB symptoms are at their lowest (Irey, personal communication; McCollum, unpublished results; Wang et al., 2006). According to Wang et al. (2006), the populations of CLas in leaves collected from field-grown 'Ponkan' mandarin and 'Shatian' pumello were highest (Ct values 28.0 to 30.2) between October and December and lowest between March and May (Ct values 32.5 to 34.8 ).

Visual incidence of HLB in the trees surveyed was calculated by summing the number of trees visually rated 1 (suspect) or 2 (likely) HLB-symptomatic and dividing by the total number of trees. The relationship between HLB incidence and CLas titer, based on either cultivar or grove, showed a strong positive correlation (Fig. 1A-B, showing HLB incidence correlated with $\mathrm{Ct}$ ); however, the correlation between grove incidence and CLas titer was stronger $\left(r^{2}=0.85\right.$ for HLB incidence and $\mathrm{Ct}$ ) than was the correlation between cultivar incidence and CLas titer $\left(r^{2}=0.54\right.$ for HLB incidence and $\mathrm{Ct}$ ) when all scions were considered. If 'Sunburst' is eliminated, the correlation between cultivar incidence and CLas titer increases $\left(r^{2}=0.85\right.$ for HLB incidence and $\mathrm{Ct}$ ). The relationship between CLas titer and HLB incidence in 'Sunburst' is unusual in that visually determined HLB incidence was fairly high $(\approx 50 \%)$ but CLas titer was low (mean $\mathrm{Ct} \approx 39$, which is $\approx 71$ CLas genome/g of tissue). One reason for this anomaly may be that the foliar symptoms suggesting HLB in 'Sunburst' may not be HLB symptoms, but may instead be nutritional deficiencies, which are frequently mistaken for HLB symptoms (Bove, 2006). Another 

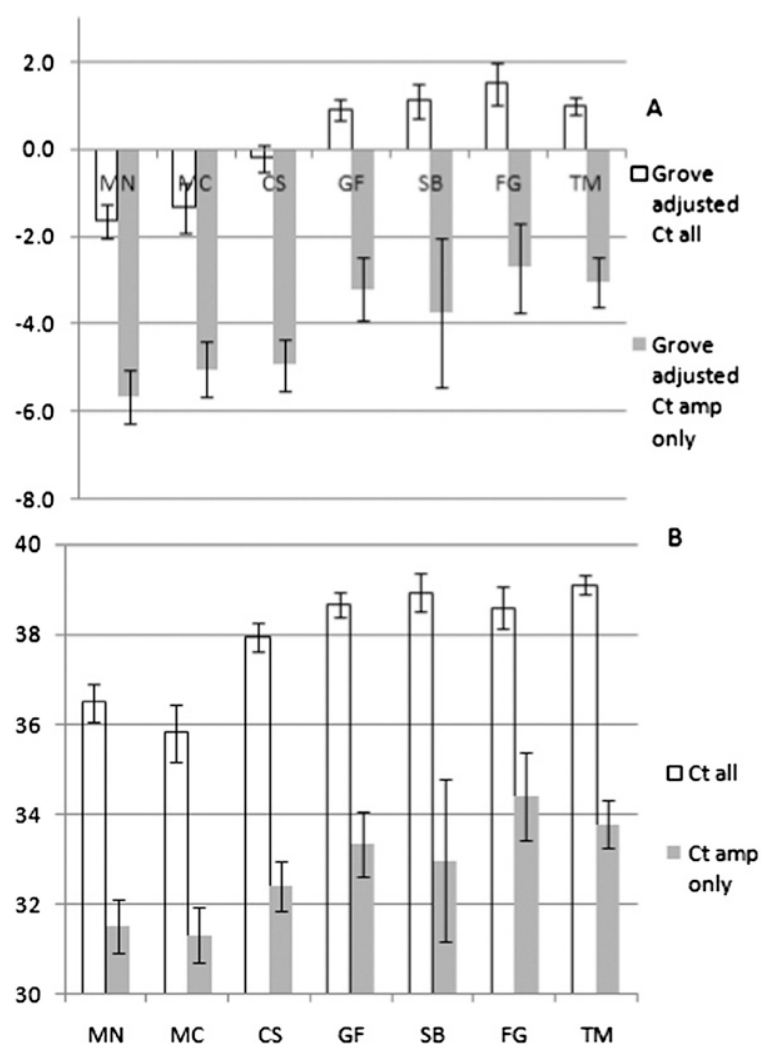

Fig. 2. Comparison of Candidatus Liberibacter asiaticus titer between cultivars in a survey of 720 trees of seven cultivars in eight groves in Florida's Indian River region in April of 2010. Ct values are directly from quantitative polymerase chain reaction for Candidatus Liberibacter asiaticus 16S rDNA. (A) Adjusted $\mathrm{Ct}$ is a grove-normalized assessment in which the average $\mathrm{Ct}$ for each grove was subtracted from each tree's Ct value. "Grove-adjusted Ct all" presents these data for all trees sampled with a Ct of 40 assigned if no amplification occurred. For "grove-adjusted $\mathrm{Ct}$ amp only," samples with no amplification were excluded. (B). "Ct all" presents data for all trees sampled with a Ct of 40 assigned if no amplification occurred. For "Ct amp only," samples with no amplification were excluded. Letters on bars indicate cultivar identity $(\mathrm{CS}=$ Citrus sinensis; FG = 'Fallglo'; GF = grapefruit; $\mathrm{MN}=$ 'Minneola'; MC = 'Murcott'; SB = 'Sunburst'; TM = 'Temple').

Table 2. Probability values for differences in CLas titer present in different cultivars (or groves) as assessed by quantitative polymerase chain reaction amplification and associated $\mathrm{Ct}$ values. ${ }^{\mathrm{z}}$

\begin{tabular}{|c|c|c|c|}
\hline \multirow[b]{2}{*}{ Sources compared } & \multirow[b]{2}{*}{ Data subset } & \multicolumn{2}{|c|}{$P$ value from nonparametric tests } \\
\hline & & CLas titer-all & $\begin{array}{l}\text { CLas titer only } \\
\text { when amplified }\end{array}$ \\
\hline All (unbalanced) & Groves all (unbalanced) & $<0.0001$ & 0.0076 \\
\hline $\begin{array}{l}\text { CS versus GF versus } \\
\text { MN versus TM }\end{array}$ & Groves $1,4,6,7,8$ & $<0.0001^{\mathrm{y}}$ & $0.0674^{x}$ \\
\hline Groves $1,4,6,7,8$ & $\begin{array}{l}\text { CS versus GF versus } \\
\text { MN versus TM }\end{array}$ & $<0.0001$ & 0.4167 \\
\hline CS versus GF & Groves $1,2,4,6,7,8$ & 0.118 & 0.162 \\
\hline $\mathrm{CS}$ versus $\mathrm{MC}$ & Groves $2,4,5$ & 0.0298 & 0.0265 \\
\hline CS versus MN & Groves $1,4,5,6,7,8$ & 0.1542 & 0.0113 \\
\hline CS versus TM & Groves $1,4,5,6,7,9$ & $<0.0001$ & 0.3605 \\
\hline $\mathrm{CS}$ versus $\mathrm{SB}$ & Groves $1,2,7$ & 0.1664 & 0.3709 \\
\hline GF versus $\mathrm{MN}$ & Groves $1,3,4,6,7,8$ & $<0.0001$ & 0.0665 \\
\hline GF versus TM & Groves $1,3,4,6,7,8$ & 0.4376 & 0.0197 \\
\hline GF versus $\mathrm{SB}$ & Groves $1,2,7$ & 0.102 & 0.2882 \\
\hline $\mathrm{MN}$ versus TM & Groves $1,3,4,5,6,7,8$ & $<0.0001$ & 0.0039 \\
\hline
\end{tabular}

${ }^{2}$ Nonparametric analyses (Kruskal-Wallis when comparing more than two data sources and Wilcoxon for comparison of two data sources in a one-sided $t$ test approximation) were conducted for each balanced comparison where cultivars were present in three or more groves. Because differences between groves were marked, only one unbalanced analysis was conducted and this compared all cultivars in all groves. For "CLas titer all," a Ct of 40 was assigned if no amplification occurred, but these samples were excluded when comparing "CLas titer only when amplified." $\mathrm{n}=20$ for each grove $\times$ scion combination. Scion types are designated as follows: $\mathrm{CS}=$ Citrus sinensis; $\mathrm{FG}=$ 'Fallglo'; $\mathrm{GF}=$ grapefruit; $\mathrm{MN}=$ 'Minneola'; $\mathrm{MC}=$ 'Murcott'; SB = 'Sunburst'; TM = 'Temple'.

${ }^{\mathrm{y}}$ By Wilcoxon one-sided $t$ test means comparison at $P \leq 0.05$, TM a; GF b; CS c; MN c. ${ }^{\mathrm{x}}$ By Wilcoxon one-sided $t$ test means comparison at $P \leq 0.06, \mathrm{TM}$ a; GF a; CS a; MN c. CLas $=$ Candidatus Liberibacter asiaticus. possible explanation for 'Sunburst' varying markedly from other cultivars in relationship to visual HLB assessment and CLas titer may be that only low levels of CLas develop even in symptomatic leaves in this cultivar.

Relevance of results to commercially significant Huanglongbing resistance. Shortterm experiments assessing differences in susceptibility to HLB among citrus cultivars are difficult to extrapolate to commercial significance. Most studies have used small potted trees in the greenhouse inoculated either by grafting (Coletta-Filho et al., 2010; Folimonova, et al., 2009; Tatineni et al., 2008) or ACP (numerous studies underway). These greenhouse studies differ markedly from natural field infections. CLas is never known to be grafttransmitted in normal commercial production and potential aspects of resistance and resulting disease progression may be very different from pathogen transmission by ACP. Use of ACP to inoculate plants in the greenhouse more closely mimics transmission in the field but may also differ in ways that influence disease development. Evaluation of trees growing in the field in the presence of HLB and the ACP vector seems to represent the most horticulturally relevant approach to assessing differences in cultivar HLB susceptibility. In this study, using existing plantings with natural exposure to HLB allows for the evaluation of mature trees immediately rather than after the several years that would be required to establish field trials, allow trees to grow, become infected, and eventually express disease symptoms. Our results confirmed that there were apparent differences in HLB incidence and CLas titer among the citrus cultivars included in the study (Fig. 1; Table 2) and that visual ratings of HLB symptoms correlated with CLas abundance (Table 3). However, although we could detect variation in HLB incidence and CLas abundance among the cultivars included in this study, these results cannot be interpreted as conclusive evidence of economically meaningful differences in susceptibility to HLB.

CLas is vectored by Asian citrus psyllids and the biology of diverse interactions among the citrus host, bacterial pathogen, and insect vector may influence disease development. Differences in tree age, tree size, flushing pattern, and coincidence among flushes, pest control, and ACP outbreaks may have all influenced these results and contributed to cultivar responses, although it is unlikely that these effects would synchronize across groves. There are anecdotal reports of lower ACP incidence in some of the cultivars showing lower HLB and CLas incidence in this study, and it is possible that increased ACP feeding on a given tree can increase the speed at which CLas titer and visual symptoms increase. A field planting of diverse citrus cultivars has been established at the USHRL Ft. Pierce farm to further test apparent cultivar difference in HLB susceptibility in a randomized complete block experiment, which will include observations on ACP feeding and development as influenced by scion cultivar.

Evaluation of HLB susceptibility through replicated field plots will require years to complete, and selection of promising cultivars 
Table 3. Ct values and Candidatus Liberibacter asiaticus genome equivalents per gram fresh weight of tissue as a function of HLB severity ratings of whole trees or diagnostic leaf samples among all groves and cultivars.

\begin{tabular}{|c|c|c|c|c|c|c|c|}
\hline \multicolumn{4}{|c|}{ Whole tree } & \multicolumn{4}{|c|}{ Leaf sample } \\
\hline \multirow[b]{2}{*}{ HLB severity ${ }^{z}$} & \multicolumn{2}{|c|}{$\mathrm{Ct}$} & \multirow{2}{*}{$\begin{array}{l}\text { Genomes per } \\
\text { gram tissue }\end{array}$} & \multirow[b]{2}{*}{ HLB severity } & \multicolumn{2}{|c|}{$\mathrm{Ct}$} & \multirow{2}{*}{$\begin{array}{l}\text { Genomes pe } \\
\text { gram tissue }\end{array}$} \\
\hline & Mean & SD & & & Mean & SD & \\
\hline 0 & 39.4 & 2.03 & 55 & 0 & 39.6 & 1.67 & 49 \\
\hline 1 & 36.5 & 4.66 & 336 & 1 & 37.8 & 3.63 & 150 \\
\hline 2 & 32.3 & 4.69 & 4576 & 2 & 32.8 & 4.94 & 3353 \\
\hline
\end{tabular}

${ }^{\mathrm{z}} \mathrm{HLB}$ severity was rated based on visible symptoms using a 3-point scale: $0=$ no apparent HLB symptoms 1 = suspect HLB symptoms; and 2 = likely HLB symptoms. Ct values were determined using quantitative polymerase chain reaction and genomes per gram tissue calculated as described in "Materials and Methods." HLB $=$ Huanglongbing.

through this report should help ensure meaningful comparisons. Quantifying HLB susceptibility has several components with only a few assessed in the short-term study reported here. CLas titers are typically high in HLBsymptomatic tissues (Coletta-Filho et al., 2010; Folimonova et al., 2009; Trivedi et al., 2009) perhaps making visual ratings most useful for such trees. However, asymptomatic tissues frequently have detectable CLas (Coletta-Filho et al., 2010; Trivedi et al., 2009), and the effect of such levels on tree productivity is unknown, reinforcing the value of qPCR to assess the rate of CLas development in evaluating susceptibility. For commercial relevance, survival and growth of young trees are critical aspects of HLB susceptibility, and the ultimate measure is economic yield over the entire life of a planting.

In the near term, the Florida citrus industry will have greatest interest in finding HLB-resistant or -tolerant varieties that have properties similar to sweet orange or grapefruit so that familiar processed products remain available at the consumer level. Even if the HLB resistance in 'Temple' (or 'Fallglo') proves usefully higher than that of sweet orange, it is not likely that fruit from these varieties will provide a direct replacement for sweet oranges. Fortunately, the genetic backgrounds of 'Temple' and 'Fallglo' are conventional and there are many related hybrids in breeding programs that offer possible HLB resistance or tolerance with traits much more like those of sweet orange.

\section{Literature Cited}

Bayer, R.J., D.J. Mabberley, C. Morton, C.H. Miller, I.K. Sharma, P. Pfeil, S. Rich, R. Hitchcock, and S. Sykes. 2009. A molecular phylogeny of the orange subfamily (Rutaceae: Aurantioideae) using nine cpDNA sequences. Amer. J. Bot. 96:668-685.

Bove, J.M. 2006. Huanglongbing: A destructive, newly-emerging, century-old disease of citrus. J. Plant Pathol. 88:7-37.

Cheema, S.S., S.P. Kapur, and J.S. Chohan. 1982. Evaluation of rough lemon strains and other rootstocks against greening-disease of citrus. Sci. Hort. 18:71-75.

Coletta-Filho, H.D., E.F. Carlos, K.C.S. Alves, M.A.R. Pereira, R.L. Boscariol-Camargo, A.A. de Souza, and M.A. Machado. 2010. In planta multiplication and graft transmission of 'Candidatus Liberibacter asiaticus' revealed by real-time PCR. Euro. J. Plant Pathol. 126:53-60.

de Lange, J.H., A.P. Vincent, and M. Nel. 1985. Breeding for resistance to greening disease in citrus. Citrus Subtropic. Fruit J. 614:6-9.

Duan, Y.P., L.J. Zhou, D.G. Hall, W.B. Li, H Doddapaneni, H. Lin, L. Liu, C.M. Vahling, D.W. Gabriel, K.P. Williams, A. Dickerman, Y.J. Sun, and T. Gottwald. 2009. Complete genome sequence of citrus huanglongbing bacterium, 'Candidatus Liberibacter asiaticus' obtained through metagenomics. Mol. Plant Microbe Interact. 22:1011-1020.

Folimonova, S.Y., C.J. Robertson, S.M. Garnsey, S. Gowda, and W.O. Dawson. 2009. Examination of the responses of different genotypes of citrus to Huanglongbing (citrus greening) under different conditions. Phytopathology 99: 1346-1354.

Gottwald, T., M. Irey, and T. Gast. 2008. The plantation edge effect of HLB; a geostatistical analysis. Proc. Int. Res. Conf. Huanglongbing. p. 305-308. 7 Sept. 2011. <http://www. plantmanagementnetwork.org/proceedings/ irchlb/2008/presentations/IRCHLB.10.6.pdf >

Gottwald, T.R. 2010. Current epidemiological understanding of citrus huanglongbing. Annu. Rev. Phytopathol. 48:119-139.

Halbert, S.E., K.L. Manjunath, and M.W. Brodie. 2008. Large-scale distribution of Diaphorina citri Kuwayama and citrus huanglongbing in Florida. Proc. Int. Res. Conf. Huanglongbing. p. 112-115. 7 Sept. 2011. <http://www. plantmanagementnetwork.org/proceedings/irchlb/ 2008/presentations/IRCHLB.2.6.pdf>.

Keiji, Y., K. Futoshi, and O. Tsuyoshi. 2005. Location and preference of adult Asian citrus psyllid, Diaphorina citri (Homoptera: Psyllidae) on Chinese box orange jasmine, Murraya exotica L. and flat lemon, Citrus depressa. Japanese J. Appl. Ento. Zool. 49:146-149.

Kiritani, K. and H.J. Su. 1999. Papaya ring spot, banana bunchy top, and citrus greening in the Asia and Pacific region: Occurrence and control strategy. Jpn. Agr. Res. Q. 33:23-30.

Koizumi, M., M. Prommintara, G. Linwattana, and T. Kaisuwan. 1997. Epidemiological aspects of citrus huanglongbing (greening) disease in Thailand. Jpn. Agr. Res. Q. 31:205-211.

Li, W., J.S. Hartung, and L. Levy. 2006. Quantitative real-time PCR for detection and identification of 'Candidatus Liberibacter' species associated with citrus huanglongbing. J. Microbiol. Methods 66:104-115.

Nariani, T.K. 1982. Integrated approach to control citrus greening disease in India. Proc. Intl. Soc. Citric. I:471-472.

NASS. 2008. Statistics by state. 7 Sept. 2011. <http:// www.nass.usda.gov/Statistics_by_State/Florida/ Publications/Citrus/broc/0708broc.pdf>.

Schwarz, R.E., L.C. Knorr, and M. Prommintara. 1973. Presence of citrus greening and its psylla vector in Thailand. Plant Protect. Bull.-FAO 21:132-138.

Shokrollah, H., T.L. Abdullah, K. Sijam, S.N.A. Abdullah, and N.A.P. Abdullah. 2009. Differential reaction of citrus species in Malaysia to Huanglongbing (HLB) disease using grafting method. Amer. J. Agr. Biol.Sci. 4:32-38.

Srinivasan, R., M.A. Hoy, R. Singh, and M.E. Rogers. 2008. Laboratory and field evaluations of Silwet L-77 and kinetic alone and in combination with imidacloprid and abamectin for the management of the Asian citrus psyllid, Diaphorina Citri (Hemiptera: Psyllidae). Florida Ent. 91:87-100.

Tatineni, S., U.S. Sagaram, S. Gowda, C.J. Robertson, W.O. Dawson, T. Iwanami, and N. Wang. 2008. In planta distribution of 'Candidatus Liberibacter asiaticus' as revealed by polymerase chain reaction (PCR) and real-time PCR. Phytopathology 98:592-599.

Trivedi, P., U.S. Sagaram, J.S. Kim, R.H. Brlansky, M.E. Rogers, L.L. Stelinski, C. Oswalt, and N. Wang. 2009. Quantification of viable Candidatus Liberibacter asiaticus in hosts using quantitative PCR with the aid of ethidium monoazide (EMA). Eur. J. Plant Pathol. 124:553-563.

Wang, Z., Y. Yin, H.H. Hu, Q. Yuan, G. Peng, and Y. Xia. 2006. Development and application of molecular-based diagnosis for 'Candidatus Liberibacter asiaticus', the causal pathogen of citrus huanglongbing. Plant Pathol. 55:630-638. 